\title{
Evaluating infusate parameters for direct drug delivery to the brainstem: a comparative study of convection-enhanced delivery versus osmotic pump delivery
}

\author{
*Julian S. Rechberger, ${ }^{1}$ Erica A. Power, BA,,,2 Victor M. Lu, MBBS, PhD, ${ }^{1}$ Liang Zhang, PhD,1 \\ Jann N. Sarkaria, MD, ${ }^{3}$ and David J. Daniels, MD, PhD ${ }^{1,4}$ \\ 1'Department of Neurologic Surgery, Mayo Clinic; ${ }^{2}$ Mayo Clinic Graduate School of Biomedical Sciences; ${ }^{3}$ Department of \\ Radiation Oncology; and ${ }^{4}$ Department of Molecular Pharmacology and Experimental Therapeutics, Mayo Clinic, Rochester, \\ Minnesota
}

OBJECTIVE Convection-enhanced delivery (CED) and osmotic pump delivery both have been promoted as promising techniques to deliver drugs to pediatric diffuse intrinsic pontine gliomas (DIPGs). Correspondingly, the aim of this study was to understand how infusate molecular weight (MW), duration of delivery, and mechanism of delivery (CED or osmotic pump) affect volume of distribution $\left(V_{d}\right)$ in the brainstem, to better inform drug selection and delivery in future DIPG investigations.

METHODS A series of in vivo experiments were conducted using rat models. CED and osmotic pump delivery systems were surgically implanted in the brainstem, and different MW fluorescent dextran beads were infused either once (acute) or daily for 5 days (chronic) in a volume infused $\left(V_{i}\right)$. Brainstems were harvested after the last infusion, and $V_{d}$ was quantified using serial sectioning and fluorescence imaging.

RESULTS Fluorescence imaging showed infusate uptake within the brainstem for both systems without complication. A significant inverse relationship was observed between infusate $M W$ and $V_{d}$ in all settings, which was distinctly exponential in nature in the setting of acute delivery across the 570-Da to 150-kDa range. Chronic duration and CED technique resulted in significantly greater $V_{d}$ compared to acute duration or osmotic pump delivery, respectively. When accounting for $V_{i}$, acute infusion yielded significantly greater $V_{d} / V_{i}$ than chronic infusion. The distribution in CED versus osmotic pump delivery was significantly affected by infusate MW at higher weights.

CONCLUSIONS Here the authors demonstrate that infusate MW, duration of infusion, and infusion mechanism all impact the $V_{d}$ of an infused agent and should be considered when selecting drugs and infusion parameters for novel investigations to treat DIPGs.

https://thejns.org/doi/abs/10.3171/2019.10.FOCUS19703

KEYWORDS diffuse intrinsic pontine glioma; DIPG; brainstem; convection-enhanced delivery; CED; osmotic pump; molecular weight; volume of distribution

$\mathrm{D}$ IFFUSE intrinsic pontine glioma (DIPG) is an incurable malignant tumor of the brainstem that typically occurs in children. The majority of these tumors harbor the H3K27M mutation, which has led to a reclassification of mutant-positive tumors in the 2016 WHO classification of tumors of the CNS as diffuse midline gliomas, H3K27M-mutant. ${ }^{4,11}$ Besides radiation ther- apy, chemotherapeutic agents have largely been unsuccessful in impacting the overall survival of patients with DIPG. ${ }^{8,12,14}$ A major barrier to establishing effective chemotherapeutic agents is the blood-brain barrier (BBB). ${ }^{1,10,18}$ Numerous techniques have been developed to overcome this hurdle, including direct delivery of therapeutic agents via intraparenchymal cannulation and interstitial infusion,

ABBREVIATIONS BBB = blood-brain barrier; BLI = bioluminescence imaging; $\mathrm{CED}=$ convection-enhanced delivery; DIPG = diffuse intrinsic pontine glioma; FITC = fluorescein isothiocyanate; $M W=$ molecular weight; $P D X=$ patient-derived xenograft; $V_{d}=$ volume of distribution; $V_{i}=$ volume infused.

SUBMITTED August 30, 2019. ACCEPTED October 3, 2019.

INCLUDE WHEN CITING DOI: 10.3171/2019.10.FOCUS19703.

* J.S.R. and E.A.P. share first authorship of this work. 
with the most common one being convection-enhanced delivery (CED) ${ }^{3,16}$

CED is administered by convection-enhanced pressure gradients that promote a relatively homogeneous distribution of molecular agents in the target parenchyma. ${ }^{3}$ Interstitial infusion by CED to the brainstem has been proven safe and feasible in multiple animal models, and recently a phase I clinical trial in children with DIPG validated this as safe in human patients. ${ }^{9}, 17,19,23,28,31$ Post hoc analyses after CED have demonstrated that therapeutic agents achieve high concentrations in the regional parenchyma and adjacent white matter tracts, with negligible efflux in the presence of an intact BBB..$^{6,7,22,32}$ Other interstitial infusion approaches distribute infusate by other mechanisms, including osmotic pump delivery, which relies solely on osmotic gradients after catheter insertion to encourage distribution within the target tissue. ${ }^{24}$ Irrespective of mechanism, these approaches all provide direct access to the brainstem and DIPG site.

Intraparenchymal infusion therapy is a potentially useful strategy to treat DIPG, although there remain a number of questions that need to be answered for optimal clinical application. ${ }^{13,16}$ This includes infusate parameters such as molecular weight (MW), which can vary widely-from < $100 \mathrm{Da}$ for simple drugs to $>150 \mathrm{kDa}$ for antibody-drug conjugates. How the volume of distribution $\left(\mathrm{V}_{\mathrm{d}}\right)$ in the brainstem varies based on this size parameter is unknown. Furthermore, there has yet to be firm evidence confirming how differences in infusion duration and delivery mechanism (i.e., CED vs osmotic pump) affect the distribution of drugs within the brainstem. Correspondingly, the aims of this study were to ascertain how 1) infusate MW, 2) duration of delivery, and 3) mechanism of delivery affect the $\mathrm{V}_{\mathrm{d}}$ within the brainstem, to better inform clinicians and scientists how therapeutic delivery to the brainstem can be maximized.

\section{Methods}

\section{Animal Preparation}

Female Sprague-Dawley rats (mean age 6 weeks, mean weight $140 \mathrm{~g}$ ) were used in this study. All animal experiments were conducted in accordance with the NIH guidelines for the use of animals in research and approved by the Mayo Clinic Institutional Animal Care and Use Committee.

\section{Infusate Agent}

Different FDS-1G fluorescein isothiocyanate-dextran (FITC-dextran) beads of MWs 3-5, 10, 20, 70, and 150 kDa (Sigma-Aldrich), as well as free Alexa Fluor 488 of MW 570 Da (ThermoFisher Scientific), were used as infusate agents in this study. They were diluted in sterile isotonic saline in advance, and formulations were prepared on the day of administration.

\section{Experimental Design}

To model the distribution of infusate in the brainstem, all rats underwent stereotactic placement of either a CED or osmotic pump delivery system followed by infusions of different MW FITC-dextran beads at $100 \mathrm{mg}$ FITC- dextran $/ \mathrm{ml}$ saline concentration, and $1 \mathrm{mg} / \mathrm{ml}$ for free Alexa. For the CED system, infusions were performed either once (acute) or daily for 5 days (chronic). For the osmotic pump delivery system, infusions were performed continuously either over a 24-hour period (acute) or over 5 days (chronic) using the Alzet minipump system, which allows for drug delivery from an implanted subcutaneous reservoir at a continuous and constant rate. Clinical assessments were performed during setup, immediately after infusion, and again before the rats were euthanized.

\section{Infusion Setup and Delivery}

Animals were anesthetized with continuous inhalation of $2.5 \%$ isoflurane for all procedures. A 1-mm burr hole was drilled using a handheld drill (8050-N/18; Dremel) $1 \mathrm{~mm}$ posterior to the sagittal suture and $1 \mathrm{~mm}$ lateral to the left lambdoid suture, as previously described..$^{30}$ The animal was then placed in a stereotactic frame (model 502650; World Precision Instruments).

\section{Convection-Enhanced Delivery}

Using the stereotactic insertion arm, a guide cannula (Plastics One, 6-mm projection below the pedestal) was inserted into the burr hole and affixed with Super Glue. Then, a 26-gauge dummy cannula (Plastics One) was placed inside the guide cannula, and skin was closed around the cannula with 4-0 absorbable Vicryl suture. For each infusion, animals were anesthetized and placed in the stereotactic frame. The dummy cannula was removed and replaced with a 33-gauge internal cannula (Plastics One, 7-8 $\mathrm{mm}$ below the pedestal) connected to a CED pump (model UMP3; World Precision Instruments). A Smartouch Controller (model Micro2T; World Precision Instruments) connected to the CED pump was used to configure infusion settings. The 1.5 -hour infusions were performed with a total volume infused $\left(\mathrm{V}_{\mathrm{i}}\right)$ of $90 \mu \mathrm{l}$ at a constant flow rate of $60 \mu \mathrm{l} / \mathrm{hr}(1 \mu \mathrm{l} / \mathrm{min})$. For animals that received a chronic 5-day (1.5 hr/day) infusion, a total $\mathrm{V}_{\mathrm{i}}$ of $450 \mu \mathrm{l}$ at a constant flow rate of $60 \mu \mathrm{l} / \mathrm{hr}(1 \mu \mathrm{l} / \mathrm{min})$ was used, with the cannula removed after and reinserted at each infusion. This flow rate was determined by considering recommended flow rates by Souweidane and colleagues $^{19,32}$ in their DIPG models $(0.1-10 \mu \mathrm{l} / \mathrm{min})$, as well as our own experience in optimizing direct delivery in rat models.

\section{Osmotic Pump Delivery}

Using the stereotactic insertion arm, a 30-gauge cannula (7-8 $\mathrm{mm}$ projection below the pedestal) was inserted into the burr hole and affixed with Super Glue. The cannula was connected to the osmotic pump by a vinyl catheter (Alzet Brain Infusion Kit II; Durect Co.). The pump was primed per the manufacturer's instructions and implanted in a subcutaneous pocket between the scapulae. The 24hour infusions were performed with a mini-osmotic pump (model 2001D) with a mean fill volume of $215 \mu \mathrm{l}$ and a mean pumping rate of $8 \mu \mathrm{l} / \mathrm{hr}$. For 5-day infusions, osmotic pumps (model 2ML1) at a mean pumping rate of 10 $\mu \mathrm{l} / \mathrm{hr}$ and with a mean fill volume of $2183 \mu \mathrm{l}$ were used. 


\section{Feasibility in Orthotopic Xenograft Model}

To extend our findings in tumor-bearing animals, we investigated acute CED in an orthotopic patient-derived xenograft (PDX) DIPG rodent model. ${ }^{30}$ Briefly, $5 \times 10^{5}$ cells of a patient-derived, luciferase-expressing, SU-DIPGXIIIp* cell line were implanted into the pons region of an athymic rat by using previously defined coordinates. ${ }^{30} \mathrm{~A}$ hollow metal screw (Plastics One) was fixed to the burr hole during the procedure. Growth of the implanted tumor was measured weekly by bioluminescence imaging (BLI), using an IVIS Lumina imaging station (Caliper Life Science) coupled to Living Image data-acquisition software (PerkinElmer) at the following settings: $150 \mathrm{mg} / \mathrm{kg}$ luciferin injected intraperitoneally $\leq 10$ minutes before imaging with an exposure time of 300 seconds. Once the tumor had reached sufficient size, the CED guide cannula was used to replace the metal screw. The position of the guide cannula in the tumor bed was visualized with CT (X-RAD SmART; Precision X-Ray). A single 90- $\mu$ l infusion of 20,000 D FD20S-1G FITC-dextran was then delivered by $\mathrm{CED}$ at $20 \mu \mathrm{l} / \mathrm{hr}$. The animal was subsequently killed and the brain was harvested and processed in a similar fashion as the other animals in the study.

\section{Tissue Preparation}

Following animal euthanasia by carbon dioxide inhalation, brains were harvested, embedded in Tissue-Tek OCT (Sakura Fintek, USA), and flash-frozen in dry ice. Coronal sections $(20 \mu \mathrm{m} / \mathrm{section})$ were sliced with a microtome cryostat (CM1860 UV; Leica Biosystems) at $-21^{\circ} \mathrm{C}$, and every fifth section was mounted on Superfrost Plus Microscope Slides (Fisher Scientific). The slides were air-dried for 24 hours, then placed under a coverslip and stored in the dark at $4{ }^{\circ} \mathrm{C}$ until analysis. Staining for histological examination was performed using $\mathrm{H} \& \mathrm{E}$.

\section{Infusate Imaging and Analysis}

Section slides were imaged with a digital slide scanner (Axio Scan.Z1; Zeiss), and fluorescence imaging was done under ultraviolet illumination (HXP 120 V Compact Light Source; LEJ GmbH). The excitation and emission maximums for all infusates were 490 and $520 \mathrm{~nm}$, respectively. Digital photographs obtained with slides under ultraviolet illumination were recorded by an Orca flash 4.0 camera (Hamamatsu Photonics) and ZEN 2.3 imaging software (Zeiss) was used to format the images. ImagePro (Media Cybernetics) was used to quantify fluorescence per slide via a semiautomated process. $V_{d}$ was calculated as the sum of fluorescent section areas multiplied by section height $(0.1 \mathrm{~mm})$. To standardize across different MWs and timings, a $\mathrm{V}_{\mathrm{d}} / \mathrm{V}_{\mathrm{i}}$ ratio was calculated based on the total $\mathrm{V}_{\mathrm{i}}$.

\section{Statistical Analysis}

Direct statistical comparisons between groups were conducted using Student t-tests or 2-way ANOVA with Tukey's multiple comparisons test when appropriate. Linear and nonlinear regression analyses were used to explore the relationship between volume parameters and MW of infusate. Statistical tests were performed using

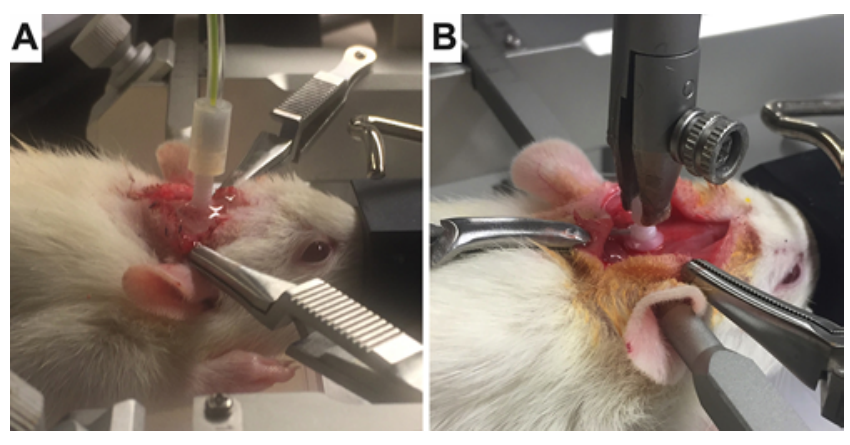

FIG. 1. Representative CED (A) and osmotic pump (B) setups of the models.

Prism 6.0 (GraphPad Software), with statistical significance set at an $\alpha$ threshold of 0.10 , and $p<0.05$ marked by an asterisk in figures.

\section{Results \\ Clinical Assessment}

To establish the safety and tolerability of CED and osmotic pump systems in vivo, we performed clinical assessments at multiples stages of setup and delivery. All delivery systems (Fig. 1) were placed and tolerated without complication. There were no procedure-related deaths, and all animals tolerated anesthesia without difficulty. We performed clinical assessments of animals after all procedures and infusions, the results of which were all unremarkable with no signs of acute or delayed neurological deficits.

\section{Histological Outcomes}

To determine the impact of CED on the brain parenchyma and confirm that there were minimal disruptive effects to the brainstem at the microscopic level following CED administration, we analyzed the brainstem histologically after CED delivery. Preliminary histological analysis was performed using standard $\mathrm{H}$ \& $\mathrm{E}$ staining of brainstems following saline-only CED (Supplementary Fig. 1). There were no obvious abnormalities in the brainstem after a single infusion (acute). After 5 daily infusions (chronic), there were histological signs of microtrauma along the cannula tract in the form of small cavitary lesions. The parenchyma surrounding the cannula tract and all nonadjacent brainstem tissue were histologically normal.

\section{Infusion Outcomes}

To better understand how infusate agents dispersed within the brainstem after direct delivery, we used fluorescence imaging of FITC-dextran beads to model distribution after infusion. We observed successful brainstem parenchymal distribution with both CED and osmotic pump delivery without visible evidence of reflux along the cannula tract (Fig. 2). In the acute CED setting fluorescence was distinct, with sharply defined borders (Fig. 2A). In comparison, acute osmotic pump delivery resulted 


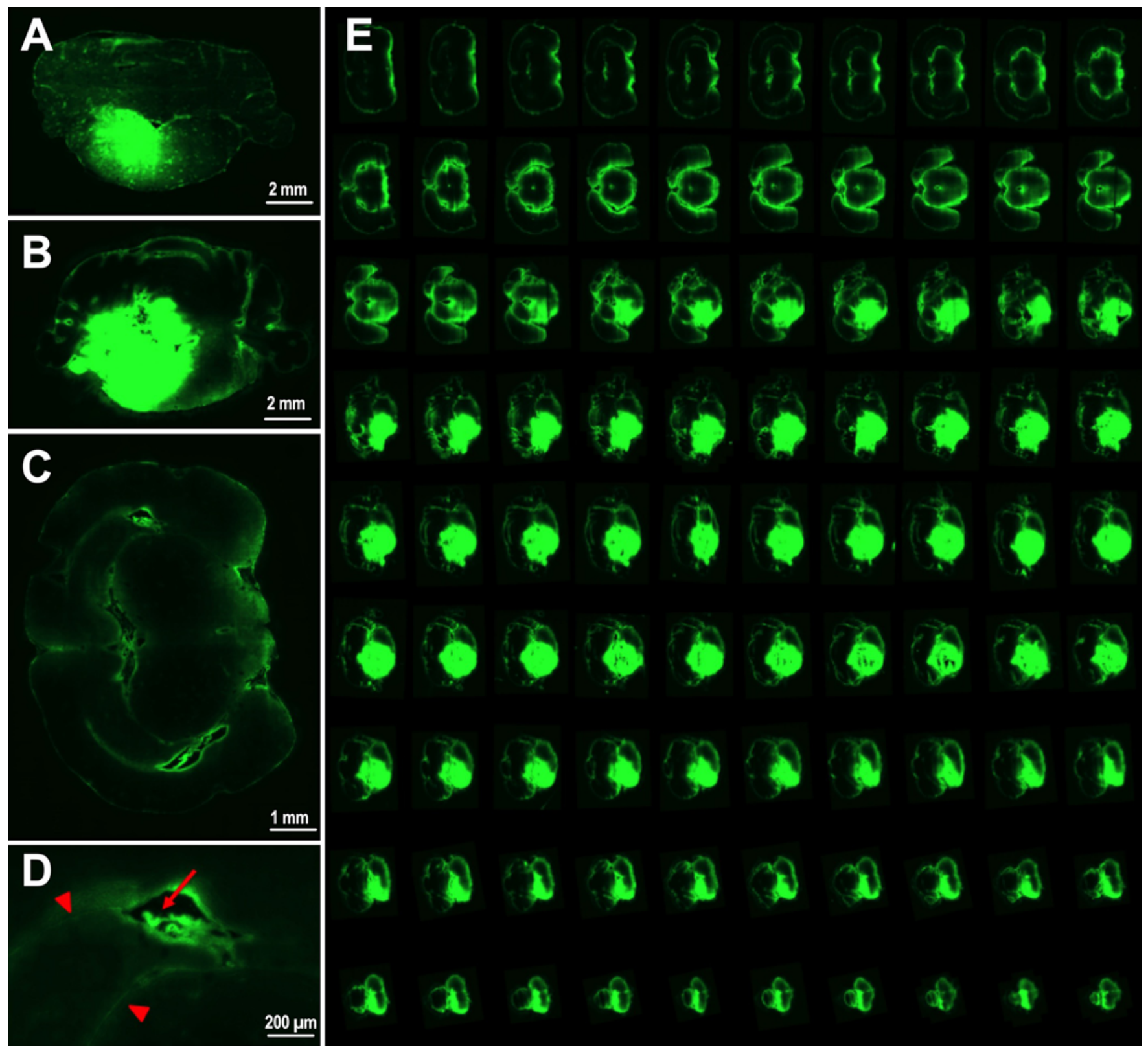

FIG. 2. Representative fluorescence (green areas) images of brainstem distribution using 10-kDa FITC-dextran beads after a single $90-\mu l$ infusion by CED (A) and 24-hour infusion by osmotic pump delivery (B). Anatomical exploration following CED in the brainstem demonstrated fluorescent parenchymal regions and blood vessels distal from the site of cannulation (C) with distribution along blood vessels (red arrow) and pial interfaces (red arrowheads) observed at higher magnification (D). A summative collage of 7-mm slices following CED with no evidence of reflux (E).

in a more heterogeneous distribution with a large zone of low fluorescence surrounding a high-fluorescence region. Moreover, there was scattered penetration around penetrating vessels of the brainstem and including the ependymal and pial interfaces (Fig. 2B). In the chronic setting, fluorescence was detected beyond the concentrated central area of infusion in a similar distribution by using either CED or osmotic pump delivery systems in the acute setting (Fig. 2C and D). Most of this distant fluorescent signal localized to the perivascular spaces, with greater paren- chymal distribution observed following osmotic pump delivery compared to CED.

\section{Effect of MW}

First, the impact of infusate MW on $V_{d}$ was examined across infusion techniques. When the analysis was limited to 3-20 kDa MW, we observed an inverse linear relationship between infusate MW and the $\mathrm{V}_{\mathrm{d}}$, irrespective of delivery system or timing: acute CED (slope $-3.1, \mathrm{R}^{2}=0.99$ ); chronic CED (slope $-2.4, \mathrm{R}^{2}=0.99$ ); acute osmotic pump 

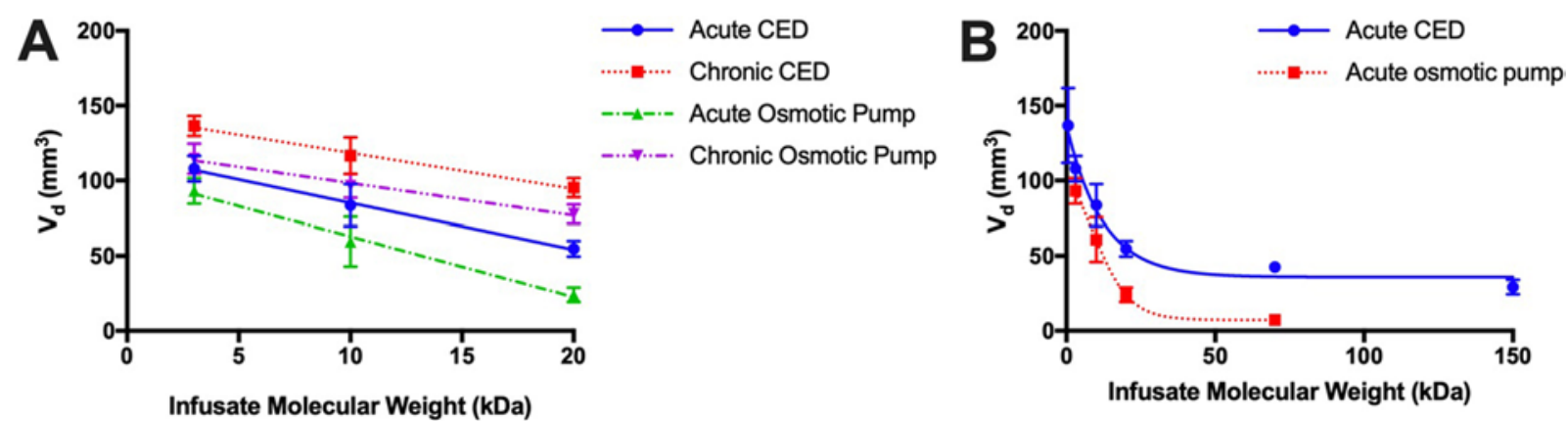

FIG. 3. Graphs showing inversely proportional relationship between infusate $M W$ and $V_{d}$ in multiple settings. $A$ : In the 3- to 20-kDa range, this relationship was linear $\left(R^{2}=0.99\right.$ in all settings). B: For acute CED and osmotic pump over a broader range, $570 \mathrm{Da}$ up to $150 \mathrm{kDa}$, this relationship was better modeled by exponential decay regression $\left(R^{2}=0.91\right.$ for acute CED and 0.96 for acute osmotic pump).

(slope -4.0, $\mathrm{R}^{2}=0.99$ ); and chronic osmotic pump (slope $-2.1, \mathrm{R}^{2}=0.99$ ) (Fig. 3A). However, when we expanded the MW range to include $570 \mathrm{Da}, 70 \mathrm{kDa}$, and $150 \mathrm{kDa}$ in the acute setting, this relationship was better fitted by an inverse exponential model instead for both acute CED $\left(\mathrm{R}^{2}=0.91\right)$ and acute osmotic pump $\left(\mathrm{R}^{2}=0.96\right)$ (Fig. 3B). These $V_{d}$ trends were reflected in the $V_{d} / V_{i}$ parameter when accounting for total volume infused (Supplementary Fig. 2A).

\section{Effect of Duration}

The next parameter we explored was delivery duration, to determine the ability of infusate agents to accumulate over time. We found that chronic delivery yielded statistically greater $V_{d}$ than acute delivery by either CED or osmotic pump within a MW range of 3-20 kDa (Fig. 4). For example, at $20 \mathrm{kDa}$ the mean $\mathrm{V}_{\mathrm{d}}$ for acute versus chronic delivery was 55 versus $95 \mathrm{~mm}^{3}(\mathrm{p}<0.01)$ using CED, and 24 versus $78 \mathrm{~mm}^{3}(\mathrm{p}<0.01)$ using an osmotic pump. However, to better account for differences in total volume being infused, we modeled the corresponding $\mathrm{V}_{\mathrm{d}} / \mathrm{V}_{\mathrm{i}}$ values. When accounting for the $\mathrm{V}_{\mathrm{i}}$, acute delivery yielded statistically greater $\mathrm{V}_{\mathrm{d}} / \mathrm{V}_{\mathrm{i}}$ than chronic delivery by both CED and osmotic pump at all corresponding MWs
(Supplementary Fig. 2B). For example, at $20 \mathrm{kDa}$ the mean $\mathrm{V}_{\mathrm{d}} / \mathrm{V}_{\mathrm{i}}$ for acute versus chronic was 0.6 versus $0.2(\mathrm{p}<0.01)$ using CED, and 0.1 versus $0.03(\mathrm{p}<0.01)$ using an osmotic pump.

\section{Effect of Delivery Mechanism}

Finally, the third parameter we sought to establish was delivery mechanism (CED vs osmotic pump). At corresponding MWs across 3-20 kDa following designated time periods, the $\mathrm{V}_{\mathrm{d}}$ following CED was statistically greater versus osmotic pump delivery at the MW of $20 \mathrm{kDa}$ only, in both acute and chronic settings: mean $\mathrm{V}_{\mathrm{d}} 55$ versus $25 \mathrm{~mm}^{3}(\mathrm{p}<0.01)$ in the acute setting, and 77 versus $18 \mathrm{~mm}^{3}$ ( $\left.\mathrm{p}<0.01\right)$ in the chronic setting (Fig. 5). Similar trends were noted when evaluating the $\mathrm{V}_{\mathrm{d}} / \mathrm{V}_{\mathrm{i}}$ parameter, although a statistically significant difference was noted between CED and osmotic pump delivery at all tested MWs (all p < 0.01) (Supplementary Fig. 2C).

\section{Feasibility in Orthotopic Xenograft Model}

To extend our findings in tumor-bearing animals, we tested acute CED of a 20-kDa infusate in a DIPG orthotopic PDX rodent model (Fig. 6A). After development of an appropriately sized pontine tumor (confirmed by BLI)
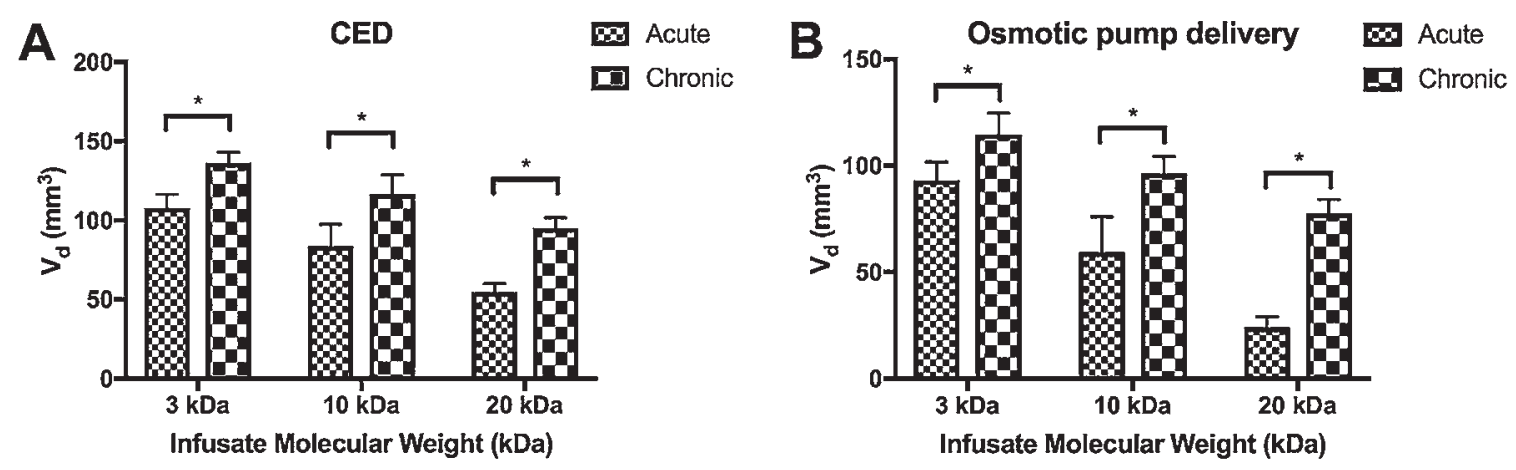

FIG. 4. Bar graphs showing comparison of $V_{d}$ after acute versus chronic infusion by $C E D(A)$ and osmotic pump delivery (B) grouped by infusate MW. * $p<0.05$. 


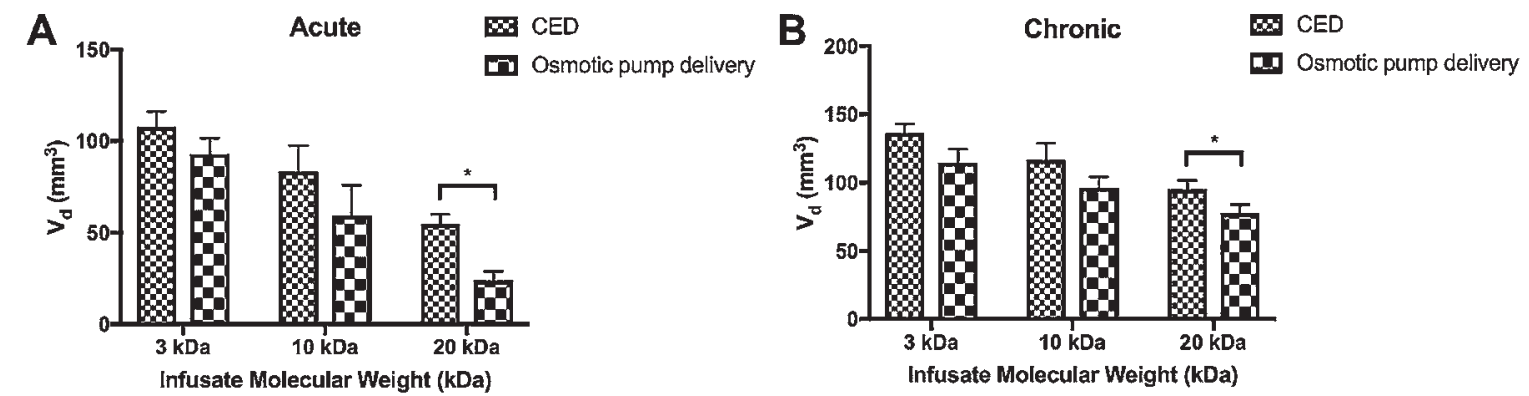

FIG. 5. Bar graphs showing comparison of $\mathrm{V}_{\mathrm{d}}$ after CED versus osmotic pump delivery infusion in either an acute setting (panel A, 1.5 hours CED, 24 hours of osmotic pump delivery) or a chronic setting (panel B, 1.5 hours CED daily for 5 days, 5 continuous days of osmotic pump delivery) grouped by infusate MW. ${ }^{*} p<0.05$.

the CED guide cannula was inserted and placement was confirmed by CT (Fig. 6B). Acute CED of a $20-\mathrm{kDa}$ infusate led to a $V_{d}$ of $63 \mathrm{~mm}^{3}$, which was largely congruent with our findings in normal brainstem delivery $\left(\mathrm{V}_{\mathrm{d}}\right.$ $\left.50.03-60.28 \mathrm{~mm}^{3}\right)$ when using the same set of infusion parameters (Fig. 6C).

\section{Discussion}

The search for effective treatment against DIPGs has not yielded much success. ${ }^{27}$ Factors that ultimately influence the effectiveness of drug therapy include intrinsic drug properties such as mechanism of action, effective delivery of the drug to the tumor, and residence time of the drug. Throughout history, one of the leading challenges in treating brain disorders is delivery of drugs across the BBB. For DIPGs this might be harder yet, because there is evidence that the blood-brainstem barrier is even more privileged. ${ }^{29} \mathrm{CED}$ is one technique that has been proposed to optimize delivery of drugs to DIPGs. Most CED studies have concentrated on the technique itself or the efficacy of the drugs being tested. Rigorous studies evaluating the physical properties of drugs used in CED like
MW, lipophilicity, and drug efflux pump liabilities have not been undertaken. The purpose of the current study was to understand the impact of infusate MW, duration of delivery, and mechanism of delivery to the brainstem on the $\mathrm{V}_{\mathrm{d}}$. Here, we demonstrate that infusate MWs ranging from $570 \mathrm{Da}$ to $150 \mathrm{kDa}$, as well as delivery duration and mechanism, all impact the intraparenchymal distribution of infusate agents in the brainstem.

The foundational in vivo efforts ${ }^{17,19,23,28,31}$ of Souweidane and colleagues have demonstrated the feasibility of interstitial infusion to the rat brainstem. In an early study, ${ }^{19}$ they validated the feasibility of delivering 20 $\mathrm{kDa}$ of FITC-dextran beads to the brainstem by means of acute CED and by chronic osmotic pump delivery. ${ }^{17}$ These earlier results mirror those observed in this study, in that both CED and osmotic pump delivery to the rat brainstem were safe. Furthermore, we were able expand and substantiate these findings across multiple infusate MWs, as well as across different durations and delivery mechanisms. In the context of targeting DIPG, the primary promise of CED and direct delivery is supported by our independent results. ${ }^{32}$
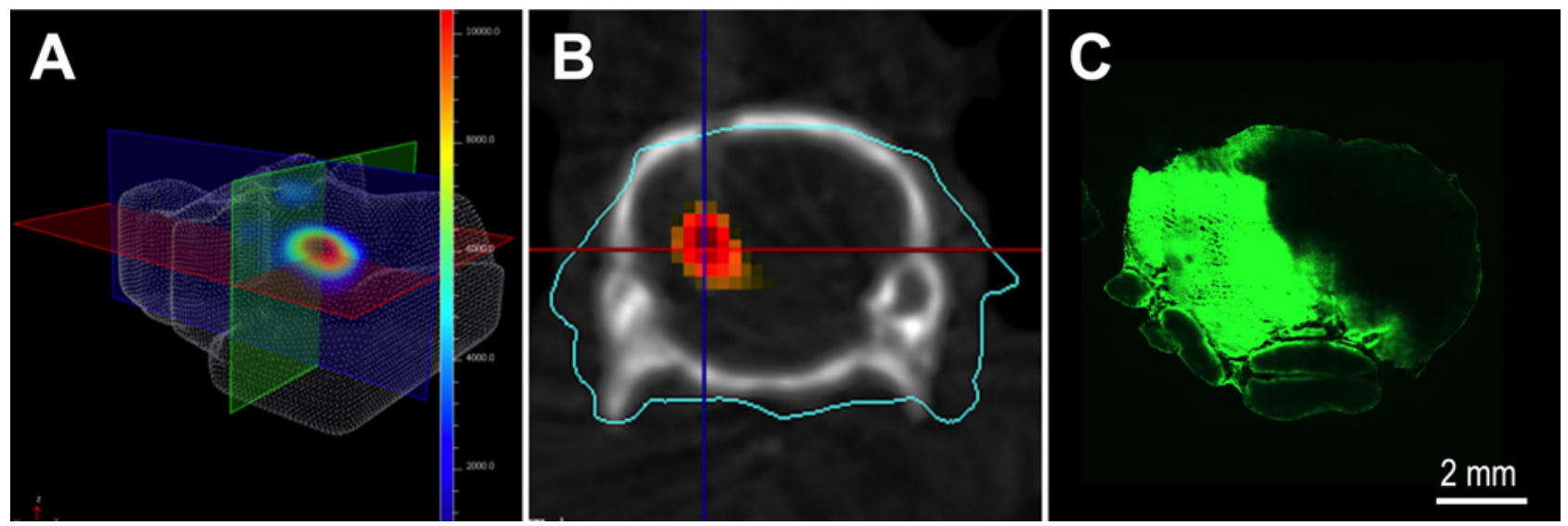

FIG. 6. Acute CED in an orthotopic PDX DIPG rodent model. A: Formation of DIPG was assessed with weekly 3D BLI. B: After the tumor had reached an appropriate size, a CED cannula was inserted and placement was confirmed by CT imaging superimposed on maximal BLI. C: Microscopic evaluation after acute CED administration of a $20-\mathrm{kDa}$ infusate confirmed $\mathrm{V}_{\mathrm{d}}$ and distribution across the entire tumor region. 
Defining how $V_{d}$ is influenced by infusate MW is crucial for optimizing direct delivery to the brain. In this study, there was an inverse relationship between infusate MW and final $V_{d}$ in the brainstem, irrespective of duration or delivery mechanism. Over a wide range of compounds from $570 \mathrm{Da}$ to $150 \mathrm{kDa}$, we found an inverse exponential relationship between infusate $\mathrm{MW}$ and $\mathrm{V}_{\mathrm{d}}$, which has implications for drug selection when choosing drugs for direct delivery to the brainstem; this includes sizes for low-MW drugs through high-MW antibody-drug conjugates. For example, in previous studies of interstitial infusion to the brainstem, a broad range of drug sizes has been used, including carmustine ${ }^{20}$ (215 Da), panobinostat ${ }^{22}$ (350 Da), and doxorubicin ${ }^{21}$ (543 Da) delivered to rats, as well as conjugated antibodies ( $>150$ $\mathrm{kDa}$ ) in the only phase I CED clinical trial ${ }^{23}$ to date targeting DIPG. Based on our findings, therapeutic agents of lower MWs would be expected to be distributed more widely in the brainstem with single injections and smaller infusion volumes.

Optimizing infusate MW was not the sole dependent variable we found in the setting of direct delivery to the brainstem. The question of duration remains a pertinent consideration in maximizing drug accumulation within the brainstem. ${ }^{15}$ In a recent study, Tosi and colleagues ${ }^{25}$ used a series of dasatinib-based drugs of varying MWs to show that infusates with lower MWs had a shorter tissue clearance half-life within the brainstem parenchyma of rats. Given the time infusates traverse across a parenchymal volume, the trends of $V_{d}$ being greater in either chronic delivery system may be better accounted for with the $\mathrm{V}_{\mathrm{d}} / \mathrm{V}_{\mathrm{i}}$ parameter, which is greater in the acute than in the chronic setting. The implications of this would be that larger proportions of infusate relative to total $\mathrm{V}_{\mathrm{i}}$ are expected in an acute setting compared to a chronic setting. The translational consequence of these trends is that the accumulation and maintenance of drug in the brainstem by direct delivery will probably require more infusions the lower the infusate MW.

A clear distinction in the literature between CED and osmotic pump delivery in the setting of the brainstem has been lacking. Although both delivery systems use infusates to infiltrate the parenchyma, they distribute within the parenchyma by means of pressure and osmotic gradients, respectively. ${ }^{3,24}$ Although the exact differences in biomechanics are beyond the scope of this article, we demonstrated that there is a significant difference between delivery methods. In direct comparison, we observed that in the higher end of our tested infusate MWs, CED resulted in a significantly greater $\mathrm{V}_{\mathrm{d}}$ than osmotic pump delivery irrespective of duration. Taken collectively, prospective studies into optimizing the delivery of drugs, especially those conjugated drugs that typically exist in the higherMW range, should carefully justify their choice of delivery mechanism to ensure maximal chance of therapeutic effect. $^{2}$

Current work in our laboratory to understand how these findings extend into DIPG models is ongoing. We found nearly similar $V_{d}$ in the setting of CED in an orthotopic DIPG PDX model when compared to our tumor-free rats when administering a $20-\mathrm{kDa}$ infusate at a single dose.
Although promising, how feasible our findings and trends are in the presence of tumor requires greater clarity. Particularly, whether or not intraparenchymal disruption in the presence of tumor affects biodistribution patterns of clinical drugs requires greater investigation in the future. ${ }^{14}$ Our findings will assist in developing a robust investigation design for these anticipated studies.

There are several limitations in this report that will benefit from future studies. First, the range of infusate MWs investigated was not expansive. Although they were sufficient to establish an inverse proportional relationship between MW and final $V_{d}$, we speculate that this relationship is only linear within a certain weight range across all possible settings. This is because our exploratory studies involving higher MWs in the acute setting, which trended toward a plateau in $\mathrm{V}_{\mathrm{d}}$ but at lower MWs, continued to increase exponentially. When testing very low-MW infusates, one avenue to explore is the possibility that lowerweight compounds diffuse out of the parenchyma too fast for effective detection. Second, our rat model is not necessarily equivalent to a human brainstem with respect to anatomical size, and therefore optimal delivery parameters may be different. For example, whereas we used a delivery rate of $1 \mu \mathrm{l} / \mathrm{min}$, clinically, delivery rates in patients with DIPG have been reported in the range of $5-10 \mu \mathrm{l} / \mathrm{min} .{ }^{9}$ Despite this, we believe the trends we report are likely to persist in patients with DIPG, although the most optimal delivery value can only truly be optimized prospectively in a clinical trial.

Another limitation for our studies is the comparability of 90 minutes of CED versus 24 hours of osmotic pump delivery to the brainstem for acute administrations, and 5 days of 90-minute injections/day for CED versus 5 days of osmotic minipump. It is difficult to directly equate CED and osmotic pump delivery biomechanics to the brainstem, and that is one reason we investigated the $\mathrm{V}_{\mathrm{d}} / \mathrm{V}_{\mathrm{i}}$ parameter in an attempt to control for any differences in volume delivered. Finally, there remain a number of other physicochemical infusate parameters to investigate to better contextualize our MW findings, including surface charge, lipophilicity, efflux pump substrates, and rates of infusion. ${ }^{5,10,32}$ If we can establish the trends that influence infusion distribution parameters, we will be better positioned to select and design novel therapies for maximum parenchymal infiltration of the brainstem, which would be desirable. Additionally, studies investigating other adjuvant therapies that may enhance further the efficacy of direct delivery to the brainstem in the setting of DIPG would be of great clinical utility. ${ }^{26}$

\section{Conclusions}

Targeting DIPGs in the brainstem has historically been hindered by drug delivery issues. Direct infusion systems such as CED present one approach that overcomes this barrier. We have demonstrated that infusate MW, duration of infusion, and infusion mechanism all impact the $V_{d}$ of an infused agent. Translationally, these findings will assist in optimization of the design of clinical drug trials and selection of drugs to treat DIPG in the brainstem by means of direct delivery. 


\section{Acknowledgments}

This work was supported by Mayo Clinic, MIT/Mayo Physical Sciences Center for Drug Distribution and Efficacy in Brain Tumors (U54 CA210180), and the foundation Humor to Fight the Tumor. Ms. Power acknowledges CTSA Grant Number UL1 TR002377 from the National Center for Advancing Translational Sciences (NCATS).

\section{References}

1. Abbott NJ, Rönnbäck L, Hansson E: Astrocyte-endothelial interactions at the blood-brain barrier. Nat Rev Neurosci 7:41-53, 2006

2. Beffinger M, Schellhammer L, Pantelyushin S, Vom Berg J: Delivery of antibodies into the murine brain via convectionenhanced delivery. J Vis Exp (149):e59675, 2019

3. Bobo RH, Laske DW, Akbasak A, Morrison PF, Dedrick RL, Oldfield EH: Convection-enhanced delivery of macromolecules in the brain. Proc Natl Acad Sci U S A 91:2076-2080, 1994

4. Castel D, Philippe C, Calmon R, Le Dret L, Truffaux N, Boddaert N, et al: Histone H3F3A and HIST1H3B K27M mutations define two subgroups of diffuse intrinsic pontine gliomas with different prognosis and phenotypes. Acta Neuropathol 130:815-827, 2015

5. El-Khouly FE, van Vuurden DG, Stroink T, Hulleman E, Kaspers GJL, Hendrikse NH, et al: Effective drug delivery in diffuse intrinsic pontine glioma: a theoretical model to identify potential candidates. Front Oncol 7:254, 2017

6. Groothuis DR, Ward S, Itskovich AC, Dobrescu C, Allen $\mathrm{CV}$, Dills C, et al: Comparison of 14C-sucrose delivery to the brain by intravenous, intraventricular, and convectionenhanced intracerebral infusion. J Neurosurg 90:321-331, 1999

7. Guisado DI, Singh R, Minkowitz S, Zhou Z, Haque S, Peck $\mathrm{KK}$, et al: A novel methodology for applying multivoxel MR spectroscopy to evaluate convection-enhanced drug delivery in diffuse intrinsic pontine gliomas. AJNR Am J Neuroradiol 37:1367-1373, 2016

8. Gwak HS, Park HJ: Developing chemotherapy for diffuse pontine intrinsic gliomas (DIPG). Crit Rev Oncol Hematol 120:111-119, 2017

9. Heiss JD, Jamshidi A, Shah S, Martin S, Wolters PL, Argersinger DP, et al: Phase I trial of convection-enhanced delivery of IL13-Pseudomonas toxin in children with diffuse intrinsic pontine glioma. J Neurosurg Pediatr 23:333-342, 2018

10. Himes BT, Zhang L, Daniels DJ: Treatment strategies in diffuse midline gliomas with the H3K27M mutation: the role of convection-enhanced delivery in overcoming anatomic challenges. Front Oncol 9:31, 2019

11. Louis DN, Perry A, Reifenberger G, von Deimling A, Figarella-Branger D, Cavenee WK, et al: The 2016 World Health Organization Classification of Tumors of the Central Nervous System: a summary. Acta Neuropathol 131:803-820, 2016

12. Lu VM, Power EA, Kerezoudis P, Daniels DJ: The 100 mostcited articles about diffuse intrinsic pontine glioma: a bibliometric analysis. Childs Nerv Syst 35:2339-2346, 2019

13. Lu VM, Rechberger JS, Himes BT, Daniels DJ: The 100 most-cited articles about convection-enhanced delivery to the brain: a bibliometric analysis. World Neurosurg 129:497502.e6, 2019

14. Lu VM, Welby JP, Mahajan A, Laack NN, Daniels DJ: Reirradiation for diffuse intrinsic pontine glioma: a systematic review and meta-analysis. Childs Nerv Syst 35:739-746, 2019

15. Matsumura $\mathrm{Y}$, Maeda $\mathrm{H}$ : A new concept for macromolecular therapeutics in cancer chemotherapy: mechanism of tumoritropic accumulation of proteins and the antitumor agent smancs. Cancer Res 46:6387-6392, 1986
16. Mehta AM, Sonabend AM, Bruce JN: Convection-enhanced delivery. Neurotherapeutics 14:358-371, 2017

17. Occhiogrosso G, Edgar MA, Sandberg DI, Souweidane MM: Prolonged convection-enhanced delivery into the rat brainstem. Neurosurgery 52:388-394, 2003

18. Pardridge WM: The blood-brain barrier: bottleneck in brain drug development. NeuroRx 2:3-14, 2005

19. Sandberg DI, Edgar MA, Souweidane MM: Convectionenhanced delivery into the rat brainstem. J Neurosurg 96:885-891, 2002

20. Sewing AC, Caretti V, Lagerweij T, Schellen P, Jansen MH, van Vuurden DG, et al: Convection enhanced delivery of carmustine to the murine brainstem: a feasibility study. J Neurosci Methods 238:88-94, 2014

21. Sewing ACP, Lagerweij T, van Vuurden DG, Meel MH, Veringa SJE, Carcaboso AM, et al: Preclinical evaluation of convection-enhanced delivery of liposomal doxorubicin to treat pediatric diffuse intrinsic pontine glioma and thalamic high-grade glioma. J Neurosurg Pediatr 19:518-530, 2017

22. Singleton WGB, Bienemann AS, Woolley M, Johnson D, Lewis $\mathrm{O}$, Wyatt MJ, et al: The distribution, clearance, and brainstem toxicity of panobinostat administered by convection-enhanced delivery. J Neurosurg Pediatr 22:288-296, 2018

23. Souweidane MM, Kramer K, Pandit-Taskar N, Zhou Z, Haque S, Zanzonico P, et al: Convection-enhanced delivery for diffuse intrinsic pontine glioma: a single-centre, doseescalation, phase 1 trial. Lancet Oncol 19:1040-1050, 2018

24. Theeuwes F, Yum SI: Principles of the design and operation of generic osmotic pumps for the delivery of semisolid or liquid drug formulations. Ann Biomed Eng 4:343-353, 1976

25. Tosi U, Kommidi H, Bellat V, Marnell CS, Guo H, Adeuyan $\mathrm{O}$, et al: Real-time, in vivo correlation of molecular structure with drug distribution in the brain striatum following convection enhanced delivery. ACS Chem Neurosci 10:2287-2298, 2019

26. Tsvankin V, Hashizume R, Katagi H, Herndon JE, Lascola $\mathrm{C}$, Venkatraman TN, et al: ABC transporter inhibition plus dexamethasone enhances the efficacy of convection enhanced delivery in $\mathrm{H} 3.3 \mathrm{~K} 27 \mathrm{M}$ mutant diffuse intrinsic pontine glioma. Neurosurgery [epub ahead of print], 2019

27. Vitanza NA, Monje M: Diffuse intrinsic pontine glioma: from diagnosis to next-generation clinical trials. Curr Treat Options Neurol 21:37, 2019

28. Wang M, Kommidi H, Tosi U, Guo H, Zhou Z, Schweitzer $\mathrm{ME}$, et al: A murine model for quantitative, real-time evaluation of convection-enhanced delivery (RT-CED) using an ${ }^{18}[\mathrm{~F}]$-positron emitting, fluorescent derivative of dasatinib. Mol Cancer Ther 16:2902-2912, 2017

29. Warren KE: Beyond the blood:brain barrier: The importance of central nervous system (CNS) pharmacokinetics for the treatment of CNS tumors, including diffuse intrinsic pontine glioma. Front Oncol 8:239, 2018

30. Welby JP, Kaptzan T, Wohl A, Peterson TE, Raghunathan A, Brown DA, et al: Current murine models and new developments in H3K27M diffuse midline gliomas. Front Oncol 9:92, 2019

31. Zhou Z, Ho SL, Singh R, Pisapia DJ, Souweidane MM: Toxicity evaluation of convection-enhanced delivery of smallmolecule kinase inhibitors in naïve mouse brainstem. Childs Nerv Syst 31:557-562, 2015

32. Zhou Z, Singh R, Souweidane MM: Convection-enhanced delivery for diffuse intrinsic pontine glioma treatment. Curr Neuropharmacol 15:116-128, 2017

\section{Disclosures}

Dr. Sarkaria received support of non-study-related clinical or research efforts that he oversaw from Novartis, Basilea, Genen- 
tech, Sanofi, Beigene, Lilly, GlaxoSmithKline, Peloton, Glionova, Bristol Myers Squibb, Cavion, Curtana, Forma, AbbVie, Actuate, Boehringer Ingelheim, Bayer, Celgene, Cible, Mitochon, and Wayshine.

\section{Author Contributions}

Conception and design: Daniels, Rechberger, Power, Zhang, Sarkaria. Acquisition of data: Daniels, Rechberger, Power, Zhang, Sarkaria. Analysis and interpretation of data: all authors. Drafting the article: all authors. Critically revising the article: all authors. Reviewed submitted version of manuscript: all authors. Approved the final version of the manuscript on behalf of all authors:

Daniels. Statistical analysis: Daniels, Rechberger, Power, Lu,

Zhang. Administrative/technical/material support: Daniels, Lu. Study supervision: Daniels.

\section{Supplemental Information}

Online-Only Content

Supplemental material is available online.

Supplementary Figs. 1 and 2. https://thejns.org/doi/suppl/ 10.3171/2019.10.FOCUS19703.

\section{Correspondence}

David J. Daniels: Mayo Clinic, Rochester, MN. daniels.david@ mayo.edu. 